\title{
FW523-3, a novel lipopeptide compound, induces apoptosis in cancer cells
}

\author{
JIAN-JUN XIE ${ }^{1 *}$, FEI ZHOU ${ }^{1 *}$, EN-MIN LI ${ }^{1}$, HONG JIANG $^{3}$, ZE-PENG DU ${ }^{2}$, \\ RU LIN ${ }^{3}$, DONG-SHENG FANG ${ }^{3}$ and LI-YAN XU ${ }^{2}$ \\ ${ }^{1}$ Department of Biochemistry and Molecular Biology; ${ }^{2}$ Institute of Oncologic Pathology, the Key Immunopathology \\ Laboratory of Guangdong Province, Medical College of Shantou University, Shantou 515041; \\ ${ }^{3}$ Fujian Provincial Key Laboratory of Screening for Novel Microbial Products, \\ Fujian Institute of Microbiology, Fuzhou 350007, P.R. China
}

Received April 13, 2011; Accepted May 16, 2011

DOI: $10.3892 / \mathrm{mmr} .2011 .496$

\begin{abstract}
FW523-3, a new lipopeptide compound, was recently isolated and purified from the culture broth of a marine Micromonospora chalcea. FW523-3 was shown to inhibit the proliferation of certain cancer cells. However, the spectra and the underlying mechanism of its antitumor activity are unclear. In this study, the MTT and colony formation assays were employed to determine the antitumor spectra of FW523-3 and its effect on cell proliferation, respectively. Apoptosis was analyzed using DNA laddering assay and flow cytometry and the involved pathways were explored by Western blotting. Results revealed that FW523-3 exhibited cytotoxicity in a panel of tumor cell lines including esophageal squamous cell carcinoma cells (EC109), lung cancer cells (A549 and 95D), gastric cancer cells (SGC7901), uterine cervix cancer cells (HeLa) and hepatocellular carcinoma cells (HepG2). Based on these results, FW523-3 inhibited the colony formation ability of tumor cells. Moreover, FW523-3 induced apoptosis via activation of caspases 9, 7 and 3 . FW523-3 also blocked the ERK and p38 signaling pathways. Taken together, we propose that FW523-3 acts as a broadspectrum antitumor drug. FW523-3 inhibits tumor cell growth and induces tumor cell apoptosis via the mitochondrial and MAPK pathways.
\end{abstract}

Correspondence to: $\mathrm{Dr} \mathrm{Li}-\mathrm{Yan} \mathrm{Xu}$, Institute of Oncologic Pathology, the Key Immunopathology Laboratory of Guangdong Province, Medical College of Shantou University, Shantou 515041, P.R. China

E-mail: liyanxu1130@yahoo.com.cn

Dr Hong Jiang, Fujian Provincial Key Laboratory of Screening for Novel Microbial Products, Fujian Institute of Microbiology, Fuzhou 350007, P.R. China

E-mail: jianghong709@yahoo.com.cn

*Contributed equally

Key words: FW523-3, antitumor bioactivity, apoptosis, mitochondrial pathway, MAPK pathway

\section{Introduction}

In 2002, an estimated 11 million new cancer cases and 7 million cancer deaths were reported worldwide, while nearly 25 million individuals were living with cancer (1). Despite the new advances in the treatment and care of cancer patients, cancer remains a major disease that defies effective cure. Currently, the majority of cancer patients are treated with chemotherapy towards the terminal stages of various types of cancer. However, a singular drawback of a number of the existing cancer chemotherapeutics is their unwanted systemic toxicity, which may result in fatality. Therefore, new therapeutics are required for effective treatment and management of this disease. New therapeutics with limited systemic toxicity, easier administration, longer efficacy and ability to act synergistically with existing chemotherapeutic drugs should not only improve the survival and quality of life of patients, but also be cost-effective. Thus, new drugs that are relevant to cancer chemotherapeutics are attractive candidates for investigation (2).

FW523-3, a lipopeptide compound, was isolated and purified from the culture broth of a marine Micromonospora chalcea (3). Its physicochemical properties and spectra revealed that FW523-3 is identical to a known antitumor antibiotic, lipopeptide rakicidin $\mathrm{B}$, which exhibited a marked cytotoxicity against the M109 cells $(3,4)$. Results of a previous study showed that FW523-3 markedly inhibits the proliferation of K562 and L929 cells and significantly reduces the size of the F-actin volume, mitochondrial quantity and membrane transmembrane potential $(3,5)$. These studies indicate that FW523-3 may have an antitumor bioactivity. However, the spectra and underlying mechanism of its antitumor activity have yet to be elucidated. In the present study, the antitumor spectrum of FW523-3 using the MTT assay was determined, and the related pathways for its antitumor activity were also investigated.

\section{Materials and methods}

Materials and cell culture. FW523-3 was isolated and purified from the culture broth of a marine Micromonospora chalcea by our team (3). Human esophageal cancer cell (EC109) and human 
Table I. Evaluation of the cytotoxicity of FW523-3 to various cell lines.

\begin{tabular}{lcccc}
\hline & \multicolumn{4}{c}{$\mathrm{IC}_{50}(\mu \mathrm{g} / \mathrm{ml})$} \\
\cline { 2 - 5 } Cells & $6 \mathrm{~h}$ & $24 \mathrm{~h}$ & $48 \mathrm{~h}$ & $72 \mathrm{~h}$ \\
\hline EC109 & $0.4470 \pm 0.0213$ & $0.4420 \pm 0.0465$ & $0.1864 \pm 0.0154$ & $0.1943 \pm 0.0098$ \\
A549 & $0.5079 \pm 0.0677$ & $0.4625 \pm 0.0177$ & $0.4105 \pm 0.0582$ & $0.4665 \pm 0.0084$ \\
95D & $0.3673 \pm 0.0319$ & $0.2396 \pm 0.0929$ & $0.2292 \pm 0.0131$ & $0.3297 \pm 0.0476$ \\
SGC7901 & $0.5374 \pm 0.0846$ & $0.4934 \pm 0.0560$ & $0.5053 \pm 0.0388$ & $0.5001 \pm 0.0335$ \\
HeLa & $0.6867 \pm 0.0263$ & $0.6088 \pm 0.0363$ & $0.4552 \pm 0.0983$ & $0.4535 \pm 0.0151$ \\
HepG2 & $0.5311 \pm 0.0114$ & $0.4711 \pm 0.1436$ & $0.3856 \pm 0.0467$ & $0.4189 \pm 0.0739$ \\
\hline
\end{tabular}
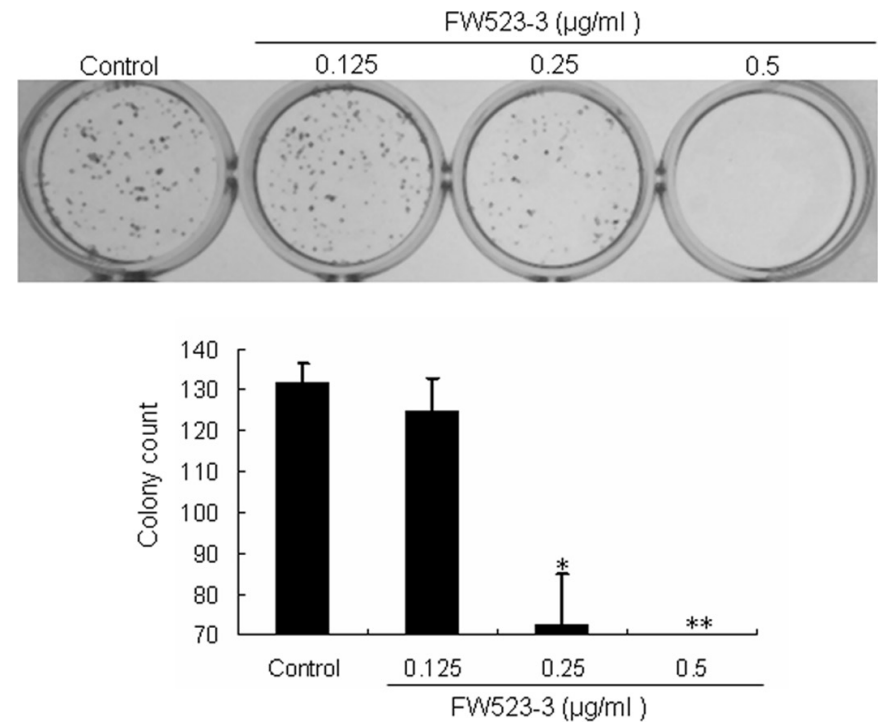

Figure 1. The effect of FW523-3 on cell colony formation. The colonyforming ability of the FW523-3-treated cells was measured ( $\mathrm{P}<0.05$; ${ }^{* *} \mathrm{P}<0.01$, compared with the control). The experiments were repeated three times, and data are presented as the means \pm SD.

uterine cervix cancer cell (HeLa) lines were cultured in 199 medium (Invitrogen, Carslbad, CA, USA) containing 10\% fetal calf serum (FCS). Human gastric adenocarcinoma (SGC7901), human hepatocellular carcinoma (HepG2), and human lung cancer (A549 and 95D) cell lines were maintained in Dulbecco's modified Eagle's medium (Invitrogen) containing 10\% FCS and F12. Cells were cultured in an atmosphere of $5 \% \mathrm{CO}_{2}$ at $37^{\circ} \mathrm{C}$.

The antibodies were used in this study: Polyclonal rabbit anti-human Fas, polyclonal rabbit anti-human ERK1/2, monoclonal mouse anti-human p-ERK1/2, monoclonal mouse anti-human p-JNK, polyclonal rabbit anti-human p38 (Santa Cruz Biotechnology, Santa Cruz, CA, USA), monoclonal mouse anti-human $\beta$-actin (Sigma, St. Louis, MO, USA), and polyclonal rabbit anti-human caspase 12 (Chemicon International). The apoptosis Sampler kit was purchased from Cell Signaling Technology.

Cytotoxicity assay. The MTT assay was used to determine the cytotoxic effect of FW523-3 (6). FW523-3 was dissolved in dimethyl sulfoxide solution (DMSO) (Sigma). Cells were seeded into 96 -well plates at a density of $1 \times 10^{4}$ cells/well (in triplicate). Various concentrations of FW523-3 were added to the medium $24 \mathrm{~h}$ later. Cells treated with equal concentration of DMSO without FW523-3 were used as controls. After incubation for $6,24,48$ and $72 \mathrm{~h}$, MTT solution $(5 \mathrm{mg} / \mathrm{ml})$ was added to the medium. The formazan crystals that formed were dissolved by DMSO, and absorption was measured at 490 and $630 \mathrm{~nm}$ with an automatic ELISA reader. The cell inhibition rate was calculated and $\mathrm{IC}_{50}(50 \%$ inhibiting concentration) was analyzed by SPSS 13.0 software. Experiments were repeated three times.

Colony formation assay. Cells were seeded into 24-well plates at a density of 100 cells/well and cultured in the medium containing FW523-3. After two weeks, cells were stained with Giemsa reagent (Sigma, Saint Louis, MO, USA), and the number of colonies ( $>50$ cells) was calculated under a microscope. The data were expressed as the means \pm SD.

Apoptosis analysis. Cells were harvested after being treated with different concentrations of FW523-3 for $48 \mathrm{~h}$ cell apoptosis was analyzed by using DNA laddering assay and flow cytometry. For DNA laddering assay, EC109 cells were lysed in buffer containing $10 \mathrm{mM}$ Tris- $\mathrm{HCl}(\mathrm{pH}$ 8.0), $0.1 \mathrm{M}$ EDTA, and $0.5 \%$ SDS for $10 \mathrm{~min}$. RNase/A and proteinase $\mathrm{K}$ were added and incubated overnight at $50^{\circ} \mathrm{C}$. The lysates were extracted with phenol and chloroform and centrifuged at $12,000 \mathrm{rpm}$ for $5 \mathrm{~min}$. DNA was precipitated in ethanol. After treatment with $1 \mathrm{M}$ Tris-HCL (pH 8.0), 0.5 M EDTA, $20 \mu \mathrm{g} /$ $\mathrm{ml}$ RNase, DNA was electrophoresed in $1.5 \%$ agarose gel and photographed.

In flow cytometry, after being treated with drugs, EC109 cells were trypsinized, washed once with PBS buffer, and fixed overnight in $70 \%$ ethanol. Fixed cells were precipitated and treated with $0.1 \%$ TritonX-100 and $0.5 \mu \mathrm{g} / \mathrm{ml}$ RNase. Cells were then centrifuged at $800 \mathrm{rpm}$ for $5 \mathrm{~min}$ and washed with PBS, and filtered through nylon mesh. Subsequently, they were incubated with $5 \mu \mathrm{g} / \mathrm{ml}$ propidium iodide (PI) (Sigma) for $30 \mathrm{~min}$ at room temperature. After washing with PBS, the stained cells were resuspended in PBS and analyzed using flow cytometry.

Western blotting. Cells were harvested after $48 \mathrm{~h}$ of treatment with FW523-3. Cell lysates were prepared in RIPA buffer [50 mM Tris-HCl, pH 8.0/150 mM NaCl/1\% (vol/vol) Nonidet 
A

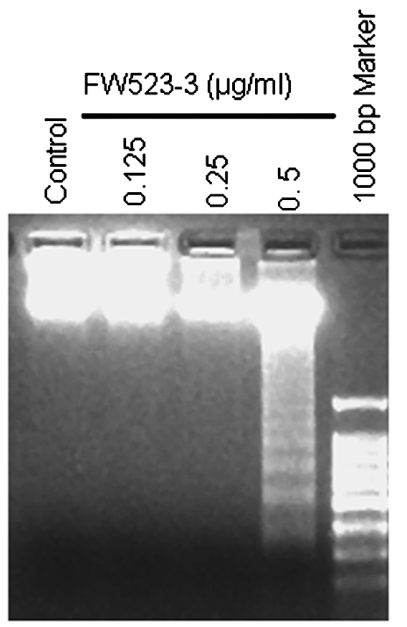

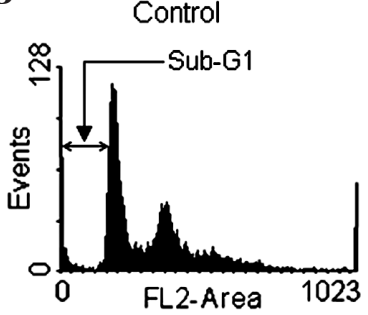

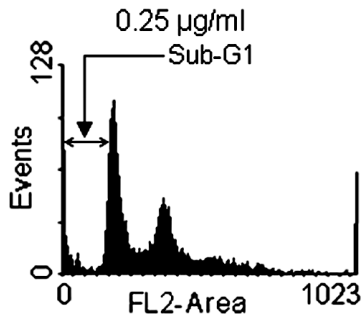

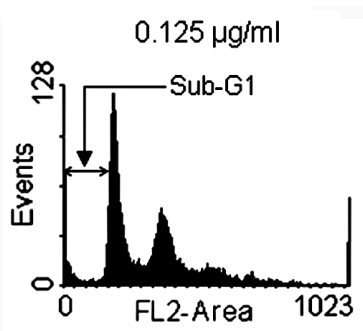

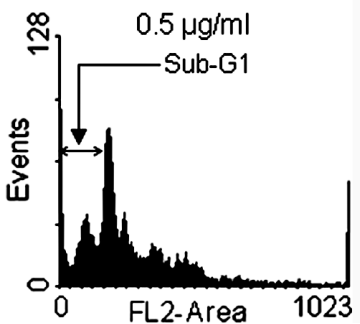

Figure 2. The effect of FW523-3 on cell apoptosis. (A) DNA laddering assay. (B) Flow cytometry results. Cells were treated with $0.125,0.25$ and $0.5 \mu \mathrm{g} / \mathrm{ml}$ FW523-3 for $48 \mathrm{~h}$ prior to harvesting. Sub-G1 was a representative of a broad hypodiploid peak.

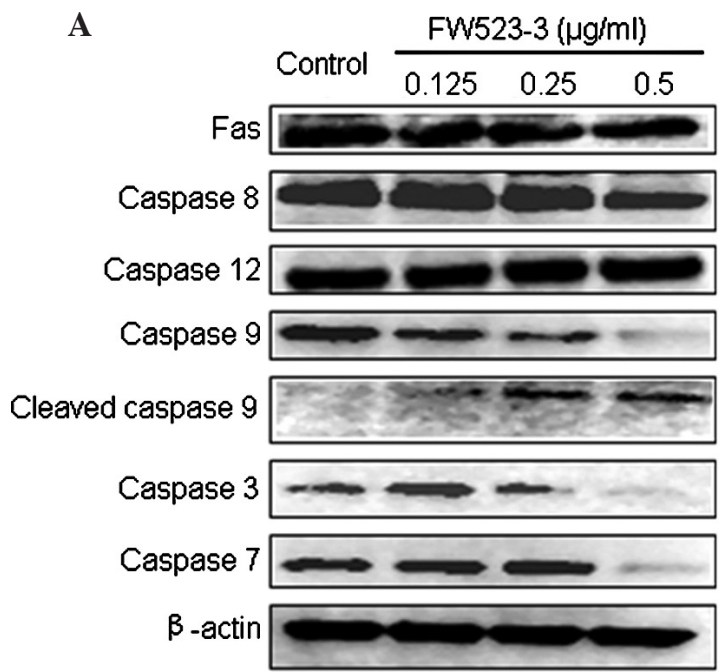

B

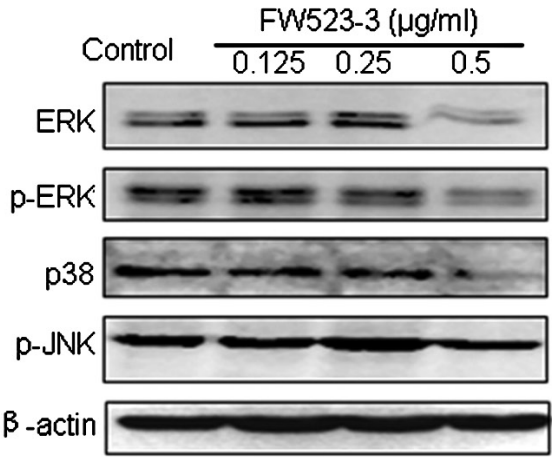

Figure 3. The effect of FW523-3 on the caspase and MAPK pathways. (A) Western blotting analysis for the changes of caspase families in FW523-3-treated cells. (B) Western blotting analysis for the expression of MAPK families in FW523-3-treated cells. Cells were treated with 0.125, 0.25 and 0.5 $\mu \mathrm{g} / \mathrm{ml}$ FW523-3 for $48 \mathrm{~h}$.

P-40/0.5\% (wt/vol) sodium desoxycholate/0.1\% (wt/vol) SDS] containing protease inhibitors, and nuclear proteins were extracted from cells according to the instructions provided with the nuclear extraction kit (Active Motif). Western blotting was performed as previously described (7). Photography and quantitative analyses of related-immunoreactive bands were carried out using the FluorChem TMIS-8900 (Alpha Innotech, Santa Clara, CA, USA).

\section{Results}

Antitumor evaluation of FW523-3 on tumor cell lines in vitro. A panel of tumor cell lines, EC109, A549, 95D, SGC7901, HeLa and HepG2, were used to evaluate the antitumor effects of FW523-3 in vitro by using MTT assay. After treatment with FW523-3 for 6, 24, 48 or 72 h, IC 50 values were detected. Results indicated that FW523-3 exhibited cytotoxicity to the tumor cell lines mentioned above (Table I). The data also indicated that FW523-3 had a higher cytotoxicity to EC109 cells $\left(\mathrm{IC}_{50}\right.$ values of EC109 cells at $48 \mathrm{~h}$ was the lowest in all values). Thus, EC109 cells were selected as the model for the subsequent studies.

Effect of FW523-3 on colony formation. A cell colony formation assay was employed to determine the bioactivity of FW523-3 on EC109 cells. Results showed that FW523-3 significantly inhibited tumor cell colony formation in a dosedependent manner. When the concentration of FW523-3 reached $0.5 \mu \mathrm{g} / \mathrm{ml}$, it almost completely inhibited colony formation (Fig. 1).

Effect of FW523-3 on apoptosis. To test whether FW523-3 killed cells by inducing apoptosis, EC109 cells were treated with FW523-3 at concentrations of $0.125,0.25$ or $0.5 \mu \mathrm{g} / \mathrm{ml}$ for $48 \mathrm{~h}$, 
and the hallmarks of apoptosis were determined. As shown in Fig. 2A, a DNA 'ladder', one hallmark of apoptosis, was apparently exhibited when EC109 cells were treated with FW523-3 at a dose of $0.5 \mu \mathrm{g} / \mathrm{ml}$. In addition to, and consistent with this result, the flow cytometry revealed that sub-G1 peak, another hallmark of apoptosis, had clearly occurred when tumor cells were treated with FW523-3 at a dose of $0.5 \mu \mathrm{g} / \mathrm{ml}$ (Fig. 2B), indicating that FW523-3 induced apoptosis of EC109 cells.

Effect of FW523-3 on the caspase cascade and MAPK pathway. To examine whether FW523-3 induced the apoptosis of EC109 cells through the activation of a caspase cascade, EC109 cells were treated with FW523-3 at 0.125, 0.25 and $0.5 \mu \mathrm{g} / \mathrm{ml}$ for $48 \mathrm{~h}$, and activation of the caspase was measured by Western blot analysis. Caspase 7 and 3 levels were significantly decreased when tumor cells were treated with FW523-3 at $0.5 \mu \mathrm{g} / \mathrm{ml}$, indicating that caspases 7 and 3 may be cleaved, as opposed to control cells in which no such changes were observed (Fig. 3A). Activation of caspases 3 and 7 can be regulated by either caspase 8 via the death receptor pathway or caspase 9 via the mitochondrial pathway (8). Therefore, we examined which pathway was predominant in the FW523-3-induced apoptosis of EC109 cells. As shown in Fig. 3A, caspase 9 was cleaved in FW523-3-treated cells, whereas caspase 8 and Fas were not changed, indicating that the mitochondrial pathway may play a significant role in this caspase cascade. Moreover, caspase 12, which mediates apoptotic signals after stress in the endoplasmic reticulum (ER) (9), was not altered. In addition, ERK and p38, two members of the MAPK family, were significantly decreased in FW523-3treated cells, indicating that the MAPK signal pathway may be involved in cell apoptosis induced by FW523-3 (Fig. 3B).

\section{Discussion}

A major obstacle to cancer chemotherapy is the development of resistant cell populations following the relapse of an initially responsive malignancy. Such chemoresistance has motivated investigators to develop new drugs that are more efficacious and have fewer side effects. A new compound, known as FW523-3, was found to have cytotoxicity to human cancer cells such as M109 (4), K562 (5) and L929 (3,5). In the present study, FW523-3 was shown to exhibit strong cytotoxicity to a panel of tumor cell lines including esophageal squamous cell carcinoma (EC109), lung cancer (A549 and 95D), gastric cancer (SGC7901), uterine cervix cancer (HeLa) and hepatocellular carcinoma (HepG2), suggesting that FW523-3 is a broad-spectrum antitumor drug. However, the effect of this compound on cell apoptosis and the underlying mechanism(s) remain unclear.

Apoptosis is a genetically controlled mechanism essential for the maintenance of tissue homeostasis, proper development and elimination of unwanted cells. In cells undergoing apoptosis, a fraction of nuclear DNA is fragmented to the size equivalent of DNA in mono- or oligonucleosomes. When such DNA is analyzed by agarose gel electrophoresis it generates the characteristic 'ladder' pattern of discontinuous DNA fragments $(10,11)$. When such apoptotic cells are stained with PI and analyzed with a flow cytometer, they exhibit a broad hypodiploid (sub-G1) peak $(10,11)$. Such a pattern of DNA degradation generally serves as a marker of the apoptotic mode of cell death. We observed that the DNA 'ladder' and sub-G1 peak clearly occurred in FW523-3-treated tumor cells, indicating that FW523-3 was capable of induce apoptosis.

The family of cysteine proteases, the so-called caspases, are critical mediators of apoptosis (9). Some of these caspases, including caspase-8, mediate signal transduction downstream of death receptors located on the plasma membrane, the so-called death receptor pathway $(12,13)$. Caspase- 9 mediate apoptotic signals after mitochondrial damage, termed the mitochondrial pathway (14). Other caspases such as caspase12 , localized to the ER and activated by ER stress, mediate an ER-specific apoptotic pathway (9). Although anticancer drugs activate the above three signaling pathways, a mitochondrial pathway is believed to play a predominant role in anticancer drug-mediated killing (15). In the mitochondrial pathway, caspase 9 is a critical protease which is activated by the release of cytochrome $\mathrm{c}$, and the activated caspase 9 leads to the activation of caspases 3, 6 and 7 and induction of apoptosis $(16,17)$. We revealed that FW523-3 activated caspases 9, 7 and 3, indicating an involvement of the mitochondrial pathway in FW523-3-induced apoptosis. In addition, numerous mechanisms exist for the regulation of the relevant MAPK pathways and various forms of cross-talk between these signal transduction pathways probably exist; the likelihood of cellular viability or death may depend on the integration of multiple signals. Xia et al proposed that the dynamic balance between growth factor-activated ERK and the stress-activated JNK-p38 pathway may be significant in determining whether a cell survives or undergoes apoptosis (18). We revealed that ERK and p38 were clearly decreased in FW523-3-treated cells, indicating that the balance of MAPK family members was broken when tumor cells were treated with FW523-3. This imbalance of MAPK members may lead to FW523-3-induced apoptosis.

In conclusion, our data revealed that FW523-3 was a broad-spectrum antitumor drug. It may induce tumor cell apoptosis via the mitochondrial and MAPK pathways.

\section{Acknowledgements}

This study was supported by grants from the National Eleventh Five-Year Plan major scientific and technological issues (no. 2009ZX9302-004), the Natural Science Foundation of China-Guangdong Joint Fund (no. U0932001) and the Science and Technology Pillar Program of Guangdong Province (no. 2009B060300016).

\section{References}

1. Kamangar F, Dores GM and Anderson WF: Patterns of cancer incidence, mortality, and prevalence across five continents: defining priorities to reduce cancer disparities in different geographic regions of the world. J Clin Oncol 24: 2137-2150, 2006.

2. Lokeshwar BL, Escatel E and Zhu B: Cytotoxic activity and inhibition of tumor cell invasion by derivatives of a chemically modified tetracycline CMT-3 (COL-3). Curr Med Chem 8: 271-279, 2001.

3. Hong J, Ru L, Wei Z and Rong CY: Purification, identification and biological activities of a lipopeptide compound FW523 from a marine Micromonospora chalcea FIM02-523. Chin J of Antibiotics (in Chinese) 31: 267-270, 2006.

4. McBrien KD, Berry RL, Lowe SE, et al: Rakicidins, new cytotoxic lipopeptides from Micromonospora sp. fermentation, isolation and characterization. J Antibiot (Tokyo) 48: 1446-1452, 1995. 
5. Hong $\mathrm{J}$ and $\mathrm{Ru} \mathrm{L}$ : In vitro antitumor activities of the rakicidin $\mathrm{B}$ FW523-3 from marine Micromonospora. Chin J of Antibiotics (in Chinese) 33: 531-533, 2008

6. Mosmann T: Rapid colorimetric assay for celluar growth and survival: application to proliferation and cytotoxicity assays. J Immunol Methods 65: 55-63, 1983.

7. Zhang H, Xu L, Xiao D, et al: Up-regulation of neutrophil gelatinase-associated lipocalin in oesophageal squamous cell carcinoma, significant correlation with cell differentiation and tumour invasion. J Clin Pathol 60: 555-561, 2007.

8. Cryns V and Yuan J: Proteases to die for. Genes Dev 12: $1551-1570,1998$

9. Nakagawa T, Zhu H, Morishima N, Li E, Xu J, Yankner BA and Yuan JY: Caspase-12 mediates endoplasmic-reticulum-specific apoptosis and cytotoxicity by amyloid-b. Nature 403: 98-103, 2000.

10. Gong J, Traganos F and Darzynkiewicz Z: A selective procedure for DNA extraction from apoptotic cells applicable for gel electrophoresis and flow cytometry. Anal Biochem 218: 314-319, 1994.

11. Carlo R and Ildo N: Analysis of apoptosis by propidium iodide staining and flow cytometry. Nature protocols 1: 1458-1461, 2006.

12. Muzio M, Chinnaiyan AM, Kischkel FC, et al: FLICE, a novel FADD-homologous ICE/CED-3-like protease, is recruited to the CD95 (Fas/APO-1) death inducing signaling complex. Cell 85: 817-827, 1996.
13. Boldin MP, Goncharov TM, Goltsev YV and Wallach D: Involvement of MACH, a novel MORT1/FADD-interacting protease, in Fas/APO-1- and TNF receptor-induced cell death. Cell 85: 803-815, 1996.

14. Li P, Nijhawan D, Budihardjo I, Srinivasula SM, Ahmad M, Alnemri ES and Wang X: Cytochrome c and dATP-dependent formation of Apaf-1/caspase-9 complex initiates an apoptotic protease cascade. Cell 91: 479-489, 1997.

15. Costantini P, Jacotot E, Decaudin D and Kroemer G: Mitochondrion as a novel target of anticancer chemotherapy. J Natl Cancer Inst 92: 1042-1053, 2000.

16. Adams JM and Cory S: The Bcl-2 protein family: arbiters of cell survival. Science 281: 1322-1326, 1998

17. Gross A, McDonnell JM and Korsmeyer SJ: BCL-2 family members and the mitochondria in apoptosis. Genes Dev 13 1899-1911, 1999.

18. Xia Z, Dickens M, Raingeaud J, Davis RJ and Greenberg ME: Opposing effects of ERK and JNK-p38 MAP kinases on apoptosis. Science 270: 1326-1331, 1995. 